\title{
Ramsey-like measurement of the decoherence rate between Zeeman sub-levels
}

\author{
M. Shuker,${ }^{1}$ O. Firstenberg, ${ }^{1}$ Y. Sagi, ${ }^{2}$ A. Ben-kish, ${ }^{1}$ N. Davidson, ${ }^{2}$ and A. Ron ${ }^{1}$ \\ ${ }^{1}$ Department of Physics, Technion-Israel Institute of Technology, Haifa 32000, Israel \\ ${ }^{2}$ Department of Physics of Complex Systems, Weizmann Institute of Science, Rehovot 76100, Israel
}

\begin{abstract}
Two-photon processes that involve different sub-levels of the ground state of an atom, are highly sensitive to depopulation and decoherence within the ground state. For example, the spectral width of electromagnetically induced transparency resonances in $\Lambda$-type system, are strongly affected by the ground state depopulation and decoherence rates. We present a direct measurement of decay rates between hyperfine and Zeeman sub-levels in the ground state of ${ }^{87} \mathrm{Rb}$ vapor. Similar to the relaxation-in-the-dark technique, pumping lasers are used to pre-align the atomic vapor in a well defined quantum state. The free propagation of the atomic state is monitored using a Ramseylike method. Coherence times in the range 1-10 ms were measured for room temperature atomic vapor. In the range of the experimental parameters used in this study, the dominant process inducing Zeeman decoherence is the spin-exchange collisions between rubidium atoms.
\end{abstract}

PACS numbers: 42.50.Gy, 32.70.Jz, 32.80.Qk, 42.50.Md

\section{INTRODUCTION}

Decay processes within the ground state strongly affect the dynamics of various two-photon processes. For example, in $\Lambda$-type electromagnetically induced transparency (EIT) [1, 2], two resonant laser fields couple two sub-levels within the ground state to a common excited state. The decoherence rate between the ground-state sub-levels, $\gamma_{12}$, determines the spectral width of the EIT, which in turn affects all related phenomena. When the EIT medium is used to reduce the group velocity of light pulses [3, 4, 5], the decoherence rate influences the minimal group velocity achievable, and, in storage of light experiments [6, 7], the decoherence rate determines the possible storage duration. In these as well as other applications of $\operatorname{EIT~(e.g.,~[8])~the~decoherence~rate~within~the~}$ ground state is a key parameter that should be carefully characterized.

Measurements of the decay rates within the ground state of various media has been extensively studied. Several methods, such as Raman scattering [9] and coherent anti-Stokes Raman scattering [10] are commonly used for decay rate measurements. Recently, an alternative method based on measuring the fluorescence from the excited state during a coherent population trapping (CPT) experiment was demonstrated [11]. In that work, the atomic medium was driven to a dark state using two lasers of orthogonal polarizations - the pump and the probe. A measurement of the fluorescence intensity from the excited level was used to calculate the decoherence rate within the ground state.

Here, we propose and demonstrate a new method, analogous to Ramsey spectroscopy, to measure the decoherence rate between Zeeman levels of the ground state. We first drive the atomic ensemble to a well known darkstate using pump and probe beams of equal intensity. Then, the driving beams are turned off and an axial magnetic field is applied to induce oscillations between the dark and the bright states of the medium. A series of weak light pulses composed of the two driving beams allows us to probe this oscillation in a non-destructive manner. By tracking the decay of the phase oscillations, the decoherence rate is directly measured. We compared the measured decoherence rate to detailed measurements we performed on the decay rate of populations between hyperfine manifolds and between Zeeman sublevels. We find that in our experiment, spin-exchange collisions are the dominant cause for decoherence between Zeeman sub-levels.

In section $\amalg$ we outline the theoretical model that was used to analyze the results of the experiments described below. In section III we describe the experimental setup. The experimental results are given in section IV] and, in section $\square$, we discuss our results and conclude.

\section{THEORETICAL MODEL}

Our experiments are performed within the D1 transition of ${ }^{87} \mathrm{Rb}$. The ground and upper states of this transition each consist of two hyperfine manifolds, $F=1,2$, which in turn contain Zeeman manifolds, as depicted in Fig. 1,B. In the experiments described below, we tune the laser to different transitions and use various polarizations. Due to the complexity of the levels structure, a proper description of the experimental results requires a model that takes into account the following elements: resonant coupling, homogenous and nonhomogenous broadening of the optical transitions, influence of spectator levels, static magnetic field, spinexchange collisions, and non-spin-preserving decay in the ground-state. Therefore, we have developed an elaborate numerical model, based on a program package (toolbox) by S. M. Tan, which was specially designed for problems in quantum optics [12].

The model describes a single ${ }^{87} \mathrm{Rb}$ atom with all sixteen sub-levels in the ground and first-excited states. It calculates the time-dependent solution of the single-atom density matrix. The coupling with the laser fields is calculated under the dipole and the rotating-wave approxi- 
mations and accounts for the various dipole moments and frequency detuning of all the allowed transitions. The parameters that govern the one-photon transitions, e.g., pressure broadening due to the buffer gas, were found from independent measurements of the absorption spectrum for the specific vapor cell used in the experiment. Doppler broadening is taken into account by performing the simulation for different velocity groups and integrating over the Boltzman distribution. Spin-exchange collisions are modeled by introducing an additional meanfield atom, with which the system interacts, presenting the density matrix of the two atoms in the product space of their electronic and nuclear spins, and assuming decoherence between the singlet and triplet states of the electronic part. This procedure is done in an iterative manner, modifying the mean-field atom according to the state of the system with each iteration. The model also include a simple linear decay mechanism which does not preserve total spin and is responsible for the decay of the system to the state of uniform population within the ground-state sub-levels (thermal equilibrium). The experimental outcome, which is the absorption coefficient in the present study, is calculated by taking the proper expectation value of the time-dependent density-matrix.

\section{EXPERIMENTAL SETUP}

The experimental setup is depicted in figure 1.A. An external cavity diode laser (ECDL) is locked to various transitions of ${ }^{87} \mathrm{Rb}$ using a saturated absorption spectroscopy setup. Different hyperfine transitions within the D1 transition of ${ }^{87} \mathrm{Rb}(\sim 795 \mathrm{~nm})$ are used for different measurements, as detailed below. The energy levels diagram of the D1 transition is depicted in figure 11,B. The intensity of the laser is controlled using an acoustooptic modulator (AOM). The first diffraction order of the AOM is used for the experiment while the zero order is sent to a beam stop. The polarization of the laser is set, either to linear or to circular, by a linear polarizer and a quarter wave-plate. The laser beam is sampled before entering the vapor cell using a beam-splitter and a detector. The beam then passes through a $5 \mathrm{~cm}$ long, 2.5 $\mathrm{cm}$ diameter vapor cell, and the transmitted intensity is measured on a second detector. By comparing the readings of the two detectors the absorption coefficient of the atomic medium is evaluated.

The vapor cell contains isotopically pure ${ }^{87} \mathrm{Rb}$ and 30 Torr of Neon buffer gas. The Rubidium vapor density in the cell is adjusted in the region $1-9 \times 10^{11} / c c$ by varying the temperature of the cell [13]. The temperature of the vapor cell is controlled using non-magnetic heaters, and stabilized to within $\pm 0.1{ }^{\circ} C$ (the heaters are switched off during the measurements). The beam waist diameter inside the vapor cell is about $6 \mathrm{~mm}$. The vapor cell is placed within a three-layered magnetic shield, which reduces the residual magnetic field in the cell to less than $50 \mu \mathrm{G}$. An axial magnetic field, $B_{z}$, is applied in some
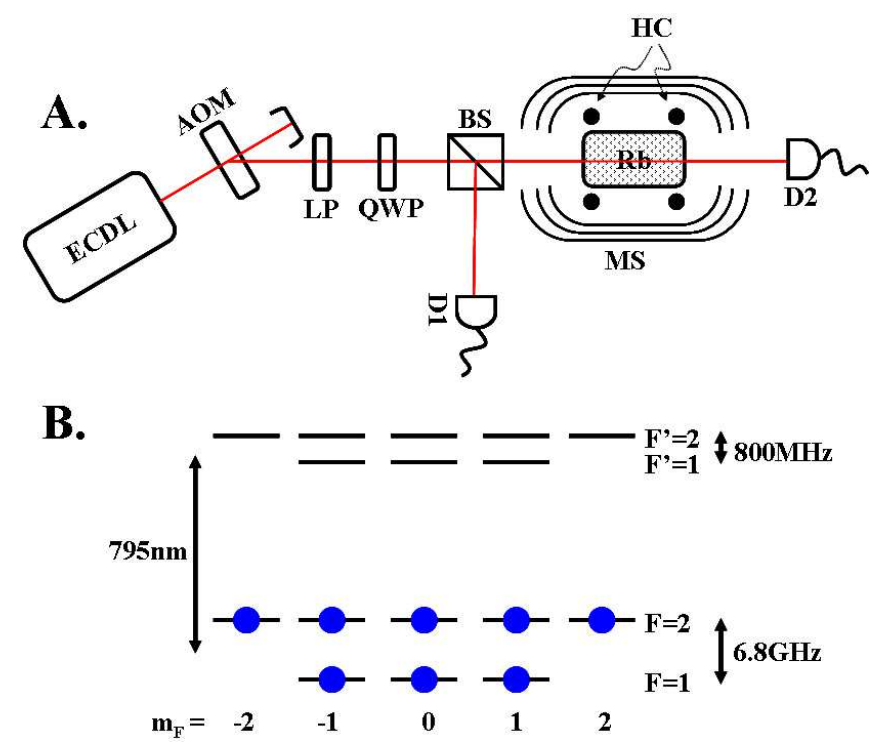

FIG. 1: (color online) A. Experimental setup: ECDL - external cavity diode laser ; AOM - acousto-optic modulator ; LP - linear polarizer ; QWP - quarter wave-plate ; MS - magnetic shield ; HC - Helmholtz coils ; D1,D2 - detectors. B. Energy levels scheme of D1 transition of ${ }^{87} R b$. Blue circles illustrate the population at thermal equilibrium.

of the measurements using a set of Helmholtz coils. This applied magnetic field sets the quantization axis, $\widehat{z}$, to be parallel to the propagation direction of the laser beams, $\widehat{k}$.

\section{EXPERIMENTAL RESULTS}

Several measurements were performed to evaluate the different decay rates within the ground state of the ${ }^{87} \mathrm{Rb}$ atom. In all the measurements a strong laser beam was used to pump the atoms to a specific quantum state. The power of the laser beam during the pumping stage was $\sim 1.5 \mathrm{~mW}$, and the pumping duration was 2 seconds (much longer than required to reach equilibrium). After the pumping stage the beam was turned off and the medium relaxed to its thermal steady-state (relaxation in the dark [14]). The relaxation rates were measured by sending a series of weak and short laser pulses ("probes") and monitoring their absorption. We typically used a power of $10 \mu \mathrm{W}$ for the probes and their duty cycle was about $5 \%$ (so the average power during the probing stage was about $500 \mathrm{nW}$ ). We verified that the probes were weak enough so their influence on the populations and coherences of the medium was negligible. By tuning the pump and the probes to various transitions and setting their polarization, different decay rates within the ground state can be measured. We first measured decay rates of populations as a reference for the main measurement of the decoherence rate between Zeeman sublevels of the 


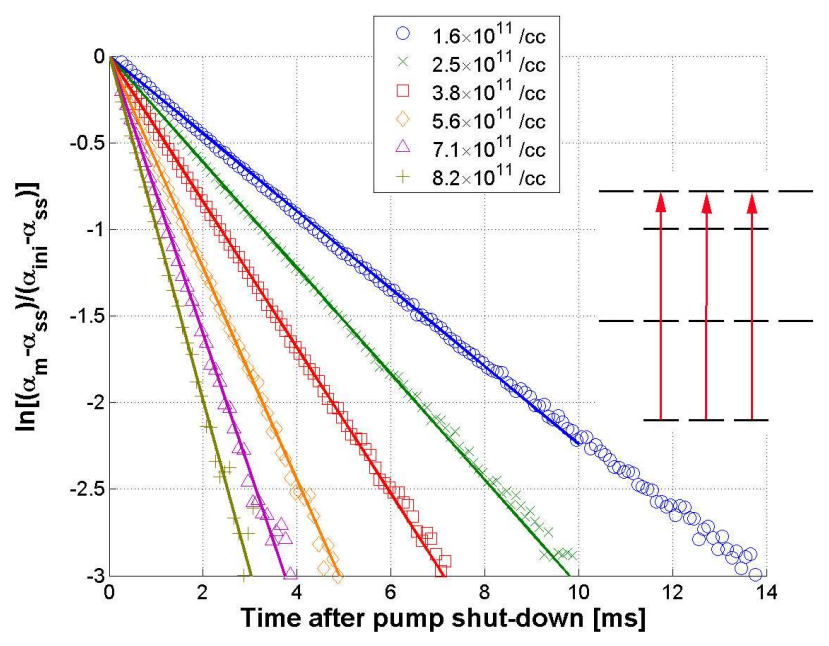

FIG. 2: (color online) Return of hyperfine population to thermal steady state after optical pumping, for different densities of the Rubidium vapor. Here $\alpha_{m}$ is the measured absorption coefficient, $\alpha_{s s}$ is the absorption coefficient at thermal steady state and $\alpha_{i n i}$ is the absorption coefficient at the end of the pumping stage. Each density shows a linear line in a semilogarithmic graph, demonstrating that the decay is exponential and the rate depends on the Rubidium density. The accuracy of determining the decay rate (i.e. the slope) was better than $5 \%$ for all the densities we measured. The inset shows the transitions driven by the laser in this measurement.

same hyperfine manifold.

\section{A. Decay of populations}

The laser is tuned to the $F=1 \rightarrow F^{\prime}=2$ transition and set to linear polarization. After the pumping stage most of the atoms populate the $F=2$ level of the ground state manifold. After the pumping process reaches a steady-state the pump beam is shut-down rapidly, and the rate of return of population to the $F=1$ level of the ground-state is monitored by measuring the absorption of the $F=1 \rightarrow F^{\prime}=2$ transition. The fraction of atoms populating the $F=1$ level is proportional to the absorption coefficient, taking into account the various ClebschGordan coefficients of the different Zeeman levels. Fig. 2 depicts the measured absorption versus time, for different rubidium densities. It is evident that the decay is exponential and that its rate depends on the rubidium density. The measured decay rate is linear with the rubidium density (see circles in Fig. 7), showing that the dominant decay mechanism of hyperfine population is Rb-Rb spinexchange collisions. From these measurements we calculated that the cross-section for Rb-Rb spin-exchange collision is $\sigma_{R b-R b}=(2.05 \pm 0.2) \times 10^{-14} \mathrm{~cm}^{2}$, in good agreement with previous measurements [15].

In order to measure the decay rate of population difference between Zeeman sub-levels, a pumping process which polarizes the medium was introduced. The pump beam was tuned to the $F=1 \rightarrow F^{\prime}=2$ transition and its polarization was set to circularly positive $\left(\sigma^{+}\right)$. A result of such a measurement is depicted in Fig. 33 alongside a reference measurement with linear polarization (one of the measurements from Fig. 21). While the linear polarization measurement shows a single exponential decay, the circular polarization measurement shows an unusual decay pattern. The population rapidly decays to higher absorption and then slowly decays down to the thermal steady-state absorption. As in the linear polarization case, the pumping process empties the $F=1$ manifold. Since the pump is circularly polarized, the steady-state population in the $F=2$ level is biased towards the higher $m_{F}$ states. After the pump shut-off, spin-exchange collisions result in rapid transfer of population back to the $F=1$ level. Since spin-exchange interaction conserves total angular momentum of the atomic ensemble, the population returning to the $F=1$ level is also biased towards higher $m_{F}$ states. Figure $3 \mathrm{~b}$ shows the populations of the three $F=1$ Zeeman sub-levels versus time, as calculated by our numerical model. When probing with the $F=1 \rightarrow F^{\prime}=2 ; \sigma^{+}$transition, the $m_{F}$ population-bias towards higher $m_{F}$ states results in higher absorption, since the Clebsch-Gordan coefficients related to the $m_{F}=-1,0,+1$ states are higher for larger $m_{F}(\sqrt{1 / 12}, \sqrt{1 / 4}, \sqrt{1 / 2}$, respectively). The slow decay of the absorption coefficient back to the steady-state absorption is the Zeeman population decay rate (decay of polarization).

Both the fast (hyperfine) and the slow (Zeeman) decays fit well an exponential decay, and hence the experimental data can be fitted well by a double exponential decay or by a single exponential decay in the case of linear polarization. The fast decay rate fits, within the experimental error, the results obtained in the linear polarization measurement, depicted in Fig. 22 The measured slow decay rate is $\sim 50 \mathrm{~s}^{-1}$, in a good agreement with the theoretical prediction of the diffusion induced decay [16], taking into account both the laser beam diameter and the vapor cell diameter. Therefore, the Zeeman population decay rate is nearly constant at different rubidium densities, as depicted in Fig. 7 (squares), and the small slope is partially due to the increased diffusion at the higher temperatures. We conclude that, in our setup, the dominant mechanism for Zeeman population decay is the diffusion of the atoms in and out of the laser beam and wall-collisions.

In other pump-probe configurations [14] it is possible to obtain a decay curve in which both the hyperfine and the Zeeman decays are in the same direction. For example, if the pump and probe are tuned to the $F=2 \rightarrow F^{\prime}=1 ; \sigma^{+}$transition, both decays increase the absorption coefficient of the medium. The configuration presented in Fig. 3 has the benefit that the fast and slow decays are at opposite directions, making the data analysis easier. We have measured the Zeeman population decay rate in both configurations and obtained similar results. 

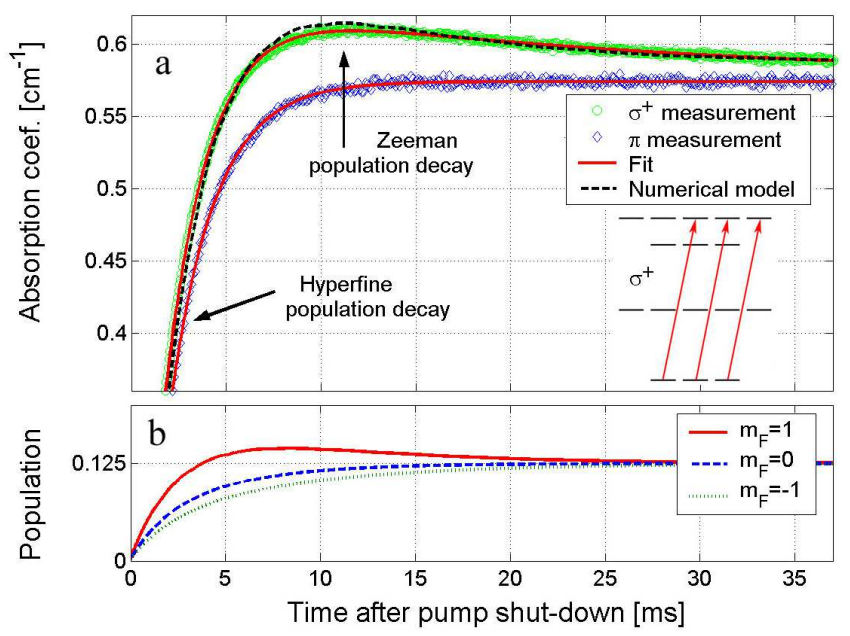

FIG. 3: (color online) (a) Decay curves, after pumping with linear and right-circular polarizations, on the $\mathrm{F}=1 \rightarrow \mathrm{F}^{\prime}=2$ transition. Both measurements were performed at a vapor density of about $3.8 \times 10^{11} / c c$ (the linear polarization measurement is one those depicted in Fig. 2). For the linear polarization (diamonds), a single exponential decay is observed due to spin-exchange. For the circular polarization (circles), a double exponential decay is observed: a fast decay towards an absorption higher than the steady-state value and a slow decay to the steady state absorption. We attribute the fast decay to hyperfine population decay and the slow decay to Zeeman population decay. The red solid lines are the respective exponential fits, while the black dashed line show the result of our numerical model. The inset shows the transitions drived by the laser in the measurement with circular polarization. (b) Results of the numerical model, showing the populations of the three Zeeman sublevels of the $\mathrm{F}=1$ manifold, during the decay. At the end of the pumping stage, the three level are nearly vacant, and they reach the equilibrium value of $1 / 8$ after the decay. Evidently, the $m_{F}=1$ level is over-populated during the fast decay, which results in an absorption higher than the steady-state value (due to the different Clebsch-Gordan coefficients).

\section{B. Decay of coherence between Zeeman sub-levels}

In this section we demonstrate a simple technique, analogous to Ramsey spectroscopy, which directly measures the decoherence rate between Zeeman sub-levels of the $F=2$ hyperfine manifold of the ${ }^{87} \mathrm{Rb}$ ground state. The first step of the measurement is to create a coherent superposition of the relevant Zeeman sub-levels. For that purpose the medium is driven on the $F=2 \rightarrow F^{\prime}=1$ transition with $\sigma^{+}$and $\sigma^{-}$polarizations with equal intensities and a well defined relative phase. The atomic medium is optically pumped until it reaches a steady state. The quantum state of the atomic system under the influence of the pumping lasers was calculated using a semi-classical model considering all sixteen relevant energy levels. The steady state solution is comprised of an incoherent mixture of two dark-states in the $F=2$ man-

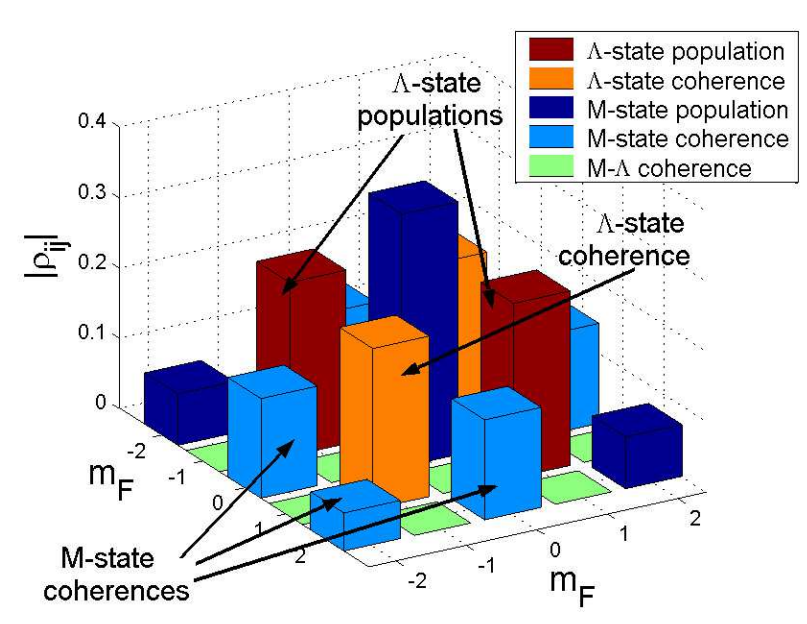

FIG. 4: (color online) Theoretical calculation, using our numerical model, of the reduced density matrix for the $F=$ 2 ground-state manifold at the end of the pumping stage (steady-state solution). Two mixed dark states arise: the $\Lambda$-state, which involves sub-levels $m_{F}=-1,+1$, and the $M$-state, which involves sub-levels $m_{F}=-2,0,+2$. The two dark-states are equally populated.

ifold of the ground state. The, so-called, $\Lambda$-state,

$$
|\Lambda\rangle=\frac{1}{\sqrt{2}}\left(\left|m_{F}=-1\right\rangle+\left|m_{F}=+1\right\rangle\right),
$$

and the, so-called, $M$-state,

$|M\rangle=\frac{1}{\sqrt{8}}\left(\left|m_{F}=-2\right\rangle+\sqrt{6} \cdot\left|m_{F}=0\right\rangle+\left|m_{F}=+2\right\rangle\right)$.

The three levels involved in the formation of the $\Lambda$-state are marked by thick lines in the inset of Fig. 5. These two dark-states are evident in Fig. 4 which depicts the reduced density matrix of the five Zeeman sub-levels in the $F=2$ manifold. Note that in the $\Lambda$-state both sub-levels have equal populations, while in the $M$-state the $m_{F}=0$ sub-level holds most of the population. This difference is due to the Clebsch-Gordan coefficients of the different optical transitions. Note that a large fraction of the atomic population accumulates in the various Zeeman sub-levels of the $F=1$ manifold (not shown in Fig. 4).

After the steady state was achieved, the pumping lasers are turned off and a small axial magnetic field, $B_{Z} \simeq$ $1 \mathrm{mG}$, is applied to the atomic medium. The magnetic field slightly shifts the energies of the Zeeman sub-levels and induces oscillations in their relative phases, at the Larmor frequency. For the special case of a dark-state with equal populations in its two lower levels (the $\Lambda$ state in our experiment), it can be easily converted to a bright state by changing the relative phase between the lower levels. The said bright-state is given by

$$
\left|\Lambda^{*}\right\rangle=\frac{1}{\sqrt{2}}\left(\left|m_{F}=-1\right\rangle-\left|m_{F}=+1\right\rangle\right) .
$$




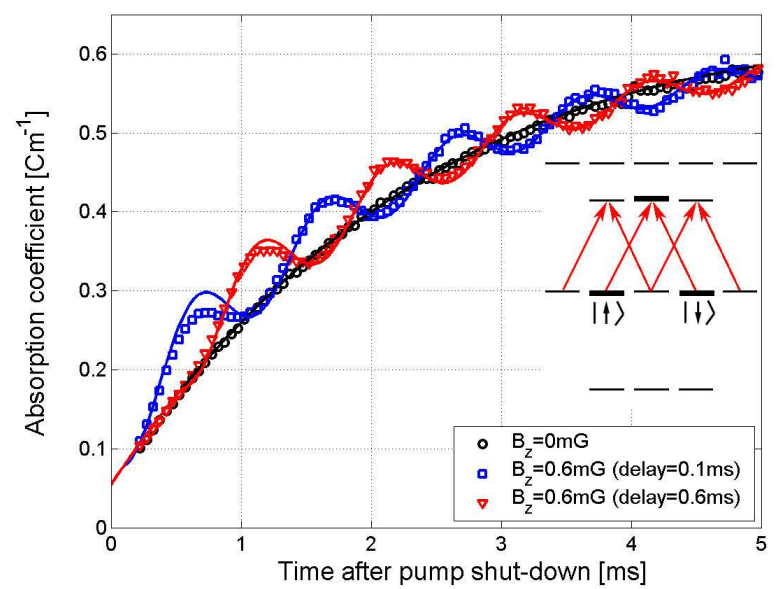

FIG. 5: (color online) Decay of the induced transparency and dark-to-bright state oscillations caused by an axial magnetic field. When no magnetic field is applied (black circles), a regular decay back to thermal absorption is observed. When an axial magnetic field is applied $0.1 \mathrm{~ms}$ after the pump shut-down (blue squares) oscillations in the absorption are observed. By applying the magnetic field after a longer delay (red triangles), the same oscillations appear at opposite phase - allowing us to extract the oscillations component of the signal with high accuracy. Solid lines show the results of our numerical model for the three cases, demonstrating a good agreement with the experimental results. An animation of the reduced density matrix for the $F=2$ manifold (as depicted in Fig. 4), during the decoherence measurement is given in [17]. The inset shows the transitions drived by the lasers in this measurement.

Hence, oscillations of the phase between the lower levels (induced by the applied axial magnetic field) results in oscillations between the dark the bright states $\left(\left|\Lambda^{*}\right\rangle\right.$ and $|\Lambda\rangle$ respectively). These 'dark-to-bright' oscillations can be detected by measuring changes in the transparency of the medium to probes composed of $\sigma^{+}$and $\sigma^{-}$with equal intensity (to which the medium is dark or bright). Decoherence between the lower levels will result in the decay of these oscillations, so decay in the amplitude of the oscillations is a direct measure of the decoherence between Zeeman sub-levels of a single hyperfine manifold. This relation was also verified by our numerical model.

For the case of a dark-state with non-equal populations in the lower levels ( $M$-state in our experiment), similar oscillations occur but with lower visibility. The amplitude of the oscillations originating from the $M$-state was calculated using our theoretical model, and should contribute about half of the total oscillation. It is interesting to note that the effect of the axial magnetic field on the $M$-state results also in very weak oscillations with twice the Larmor frequency. These oscillations are not observable in our experimental system due to their low amplitude.

Fig. 5 depicts the results of a Zeeman decoherence measurement. When no magnetic field is applied $\left(B_{Z}=\right.$ 0 ), a decay of the transparency is observed (blue circles), which occurs due to decoherence as well as other decay mechanisms. When a small, DC, magnetic field is applied $\left(B_{Z} \neq 0\right)$ after the pump is shut down, oscillations in the transparency are clearly apparent - a result of the medium's oscillations from the dark to the bright state and vice versa (green squares). Due to technical considerations, the magnetic field is switched on with a delay of $0.1 \mathrm{~ms}$ after the pump is shut-down. The cycle time of the observed oscillations is determined by the magnitude of the applied magnetic field, and their amplitude depends mostly on the magnitude of the coherence terms between Zeeman sub-levels. Immediately after the pump is shut-down, the medium is at the dark state. When the magnetic field is switched on, an increase in the absorption coefficient is observed, followed by a decrease back to the $B_{Z}=0$ curve. After a few cycles, the minima of the oscillations decrease below the $B_{Z}=0$ curve, due to a small residual magnetic field which causes slow oscillations even when no magnetic field is applied. In order to extract, with high accuracy, the oscillation component of the signal, a second measurement was performed with a longer delay between the pump shut-down and the application of the magnetic field. The delay was chosen to be about $0.6 \mathrm{~ms}$ (red triangles), so that the two measurements will have a phase-shift of $\pi$. By subtracting the two measurements, the oscillations component can be extracted from the signal, as depicted in Fig. 6. The oscillation component is well fitted by a sinusoidal function multiplied by an exponential decay whose rate is equivalent to the decoherence rate between the Zeeman levels of the $F=2$ hyperfine manifold. We have repeated this measurement for different rubidium densities, and found that the decay rate is similar to the hyperfine population decay rate (see Fig. 7 and section $\mathrm{V}$ ). By repeating the measurements with different applied magnetic filed, we have verified that the measured decoherence rate is independent of the applied field's magnitude.

The decoherence measurement presented here is analogues to the Ramsey spectroscopy method. For the purpose of illustration we consider the $\Lambda$-state, and denote its two lower levels $|\uparrow\rangle=\left|F=2 ; m_{F}=-1\right\rangle$ and $|\downarrow\rangle=\left|F=2 ; m_{F}=+1\right\rangle$ as depicted in the inset of Fig. 5. As explained above, the pumping process creates a coherent super-position of the two levels, given by $1 / \sqrt{2}(|\uparrow\rangle+|\downarrow\rangle)$. After the pumping fields are turned off, the super-position's phase is allowed to oscillate. In the present experiment, since the two levels $|\uparrow\rangle,|\downarrow\rangle$ are degenerate, a small magnetic field is applied to drive phase oscillations. After a certain time delay, the phase between the two levels is detected by sending two laser pulses to which the transparency depends on the phase of the super-position. By repeating this measurement for different time delays, a graph monitoring the phase oscillations, as well as the loss of coherence is obtained. 


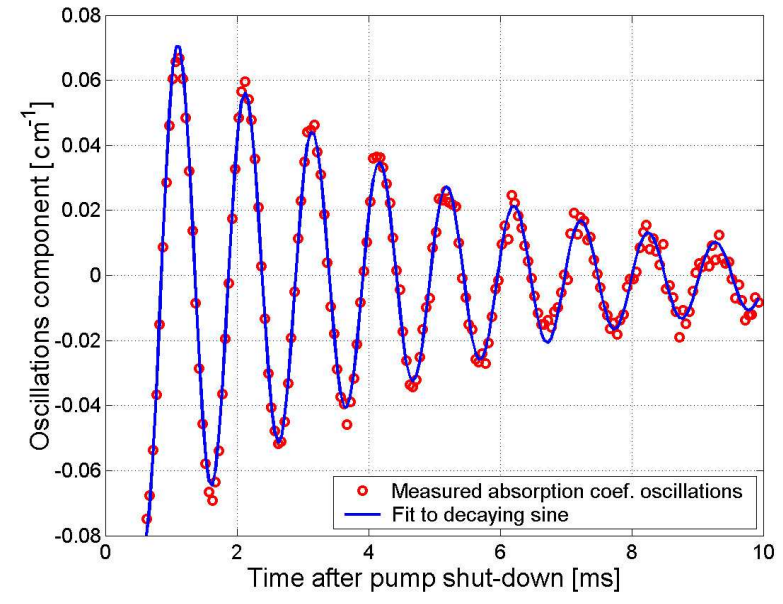

FIG. 6: (color online) The signal of dark-to-bright state oscillations, which was extracted from the data in Fig. 5. A decaying sinusoidal function fits the measured data well.

\section{DISCUSSION AND CONCLUSIONS}

We have presented measurements of various decay rates in the ground state manifold of atomic rubidium. The experimental technique is based on the relaxationin-the-dark method [14]. By tuning the laser to different transitions and polarizations, as well as switching on a magnetic field at the probing stage, we were able to measure independently the decay rates of population between hyperfine and Zeeman levels, and the decoherence rate between Zeeman sub-levels of the same hyperfine manifold. The results of all the measurements are presented in Fig. 7

The decay of populations between hyperfine levels is dominated by spin-exchange collisions between rubidium atoms, and hence depends linearly on the rubidium density. The decay of population between Zeeman sub-levels, i.e., the decay of polarization, is dominated by the diffusion of atoms in the vapor cell, and is nearly independent of the rubidium density. The decoherence rate between Zeeman sub-levels of the same hyperfine manifold is equal to the hyperfine population decay rate within the measurement accuracy. We conclude that no additional decay mechanism contributes to the decoherence rate without affecting the decay of populations. Unlike optical transitions, where the decoherence rate can be half the population decay rate [18], we find that the decoherence rate between ground-state levels is similar to the population decay rate. We attribute this to the fact that both levels are affected by the population decay.

The various decay rates within the ground state are the key parameters of any two-photon interaction, e.g., in EIT. The decay rates directly affect all the features of the EIT phenomena, such as slow light, storage of light, frequency standards, etc. Simple techniques to measure the

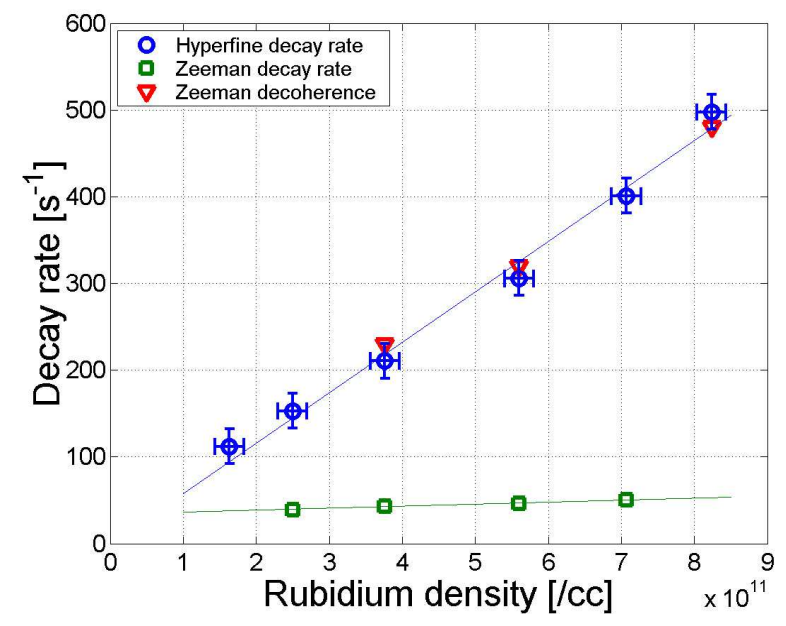

FIG. 7: (color online) Measured decay rates vs. rubidium atomic density: hyperfine population decay (blue circles), Zeeman population decay (green squares) and Zeeman decoherence (red triangles). The solid lines are a guide to the eye.

decoherence rate are important for many practical purposes and may be useful at different realizations of twophoton phenomena. In the current experiment we measured the decoherence rate between Zeeman sub-levels of the same hyperfine manifold. The same method can be applied for measuring the decoherence rate between levels belonging to different hyperfine manifolds. In addition, this method can be adapted to any medium exhibiting EIT, by applying an analogous technique to oscillate the relative phase between the lower levels.

\section{Acknowledgments}

We wish to acknowledge the help of Amnon Fisher, Yoav Erlich and Igal Levi. This research was partially supported by the fund for encouragement of research in the Technion.
[1] S. E. Harris, Physics Today 50, 36 (1997).

[2] E. Arimondo, Progress in Optics, vol. 35 (Elsevier, Amsterdam, 1996).

[3] A. Kasapi, M. Jain, G. Y. Yin, and S. E. Harris, Phys.
Rev. Lett. 74, 2447 (1995).

[4] M. M. Kash, V. A. Sautenkov, A. S. Zibrov, L. Hollberg, G. R. Welch, M. D. Lukin, Y. Rostovtsev, E. S. Fry, and M. O. Scully, Phys. Rev. Lett. 82, 5229 (1999). 
[5] L. V. Hau, S. E. Harris, Z. Dutton, and C. H. Behroozi, Nature 397, 594 (1999).

[6] C. Liu, Z. Dutton, C. H. Behroozi, and L. V. Hau, Nature 409, 490 (2001).

[7] D. F. Phillips, A. Fleischhauer, A. Mair, R. L. Walsworth, and M. D. Lukin, Phys. Rev. Lett. 86, 783 (2001).

[8] S. E. Harris and L. V. Hau, Phys. Rev. Lett. 82, 4611 (1999).

[9] W. Demtrder, Laser Spectroscopy (Springer-Verlag, New York, 2002).

[10] Y. R. Shen, Principles of Non-linear Optic (Wiley, New York, 1984).

[11] A. K. Patnaik, P. S. Hsu, G. S. Agarwal, G. R. Welch, and M. O. Scully, Phys. Rev. A 75, 023807 (2007).

[12] S. M. Tan, Journal of Optics B: Quantum and Semiclas- sical Optics 1, 424 (1999).

[13] D. A. Steck, Tech. Rep. LA-UR-03-8638, LANL (2003).

[14] W. Franzen, Phys. Rev. 115, 850 (1959).

[15] W. Happer, Rev. Mod. Phys. 44, 169 (1972).

[16] P. G. Pappas, R. A. Forber, W. W. Quivers, R. R. Dasari, M. S. Feld, and D. E. Murnick, Phys. Rev. Lett. 47, 236 (1981).

[17] See EPAPS Document No. ??? for a movie showing the dynamics of the reduced density matrix during the Zeeman decoherence measurement. For more information on EPAPS, see http://www.aip.org/pubservs/epaps.html.

[18] C. Cohen-Tannoudji, J. Dupont-Roc, and G. Grynberg, Atom-Photon Interactions : Basic Processes and Applications (Wiley-Interscience, 1998). 